\title{
IRNSS Signal Acquisition and Tracking
}

\author{
Shreyas.R ${ }^{1}$, S E Pramod ${ }^{2}$, Rajat Gupta ${ }^{3}$ \\ Alumni, Department of ECE, BNM Institute of Technology, Bangalore, India ${ }^{1,2,3}$
}

\begin{abstract}
IRNSS is an independent regional navigation satellite system being developed by India. It is designed to provide accurate position information service to users in India as well as the region extending up to $1500 \mathrm{~km}$ from its boundary, which is its primary service area. An Extended Service Area lies between primary service area and area by the rectangle from Latitude 30 degree south to 50 degree north, Longitude 30 degree east to 130 degree east. This paper is focused primarily on developing a software based receiver for the signals transmitted from IRNSS satellites. Tracking and positioning of IRNSS device is primarily preceded by acquisition stage which involves serial search and autocorrelation methods.
\end{abstract}

Keywords: IRNSS, PRN, C/A, BPSK, NCO

\section{INTRODUCTION}

IRNSS is abbreviated as "Indian Regional Navigation Satellite System" with an operational name of NAVIC (Navigation with Indian Constellation). It is an autonomous regional satellite navigation system, that provides accurate real time positioning and timing services. It covers India and region extending $1500 \mathrm{~km}$ around it, with plans for further extension. The system at present consist of a constellation of seven satellites with 3 satellites located in Geostationary orbit while remaining 4 are inclined in Geosynchronous orbit. The system was developed partly because access to foreign government controlled GLONASS is not guaranteed in hostile situations, as happened to Indian Military in 1999 when it was dependent on American GPS during Kargil war.

The IRNSS Receiver can be either software based or hardware based not in totality though. The unit which performs signal processing on the received IRNSS signals decides be it hardware based or software based. IRNSS system currently has Analog based receivers which are quite large in size making them unsuitable for installation in small devices. An approach is being made in this paper to develop software-based receiver. IRNSS software-based receiver is developed by using high level programming language MATLAB for signal processing. The major reason to switch to software-based receiver system is that software allows for switching between algorithms depending upon the received signal strength.

In this paper, we limit our discussion within the L5 band and C/A ( Coarse Acquisition) code only since S band and P code are used primarily for military purpose and the encryption codes are not available to the civilian community. We will explain briefly about GPS signal structure and software-based GPS receiver architecture. The main focus of the paper is on how GPS signal can be processed by using acquisition and tracking algorithms to extract the navigation data bits from the raw data. The navigation data bits provide all the necessary information to compute the pseudo range between the receiver and the visible satellites.

\section{PROPOSED SYSTEM}

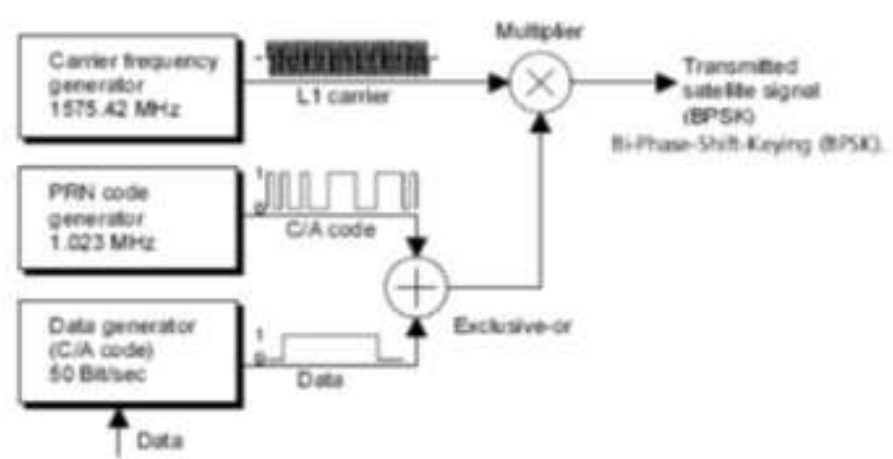

Figure 1. Simplified IRNSS SPS Modulator Configuration 
Vol. 8, Issue 4, April 2019

A simplified model of the L1 SPS modulator configuration is shown in the figure. The L1 carrier is modulated by the modulo2 sum of the C/A code and the navigation data. The modulo-2 sum is the same operation as the exclusive-OR operation. The nominal chipping rate of the C/A code is $1.023 \mathrm{MHz}$ and one code period is 1023 chips long or $1 \mathrm{~ms}$. It is important to notice that the transitions of data bits are synchronized to code periods, but at a much slower rate $(50 \mathrm{~Hz})$.

Before Binary Phase Shift Keying (BPSK) modulation is employed, the above described modulo-2 sum with values $\{0,1\}$, are mapped to $\{-1,1\}$. The transmitted L1 SPS signal is defined by

\section{$\operatorname{Li}(W 1 t)=A[C A i(t)$ Xor Di $(t)] \sin ($ W1 $t)$}

Where: Li broadcast signal from ith satellite

i indicates satellite number

A signal amplitude

CAi C/A code for ith satellite

D navigation data for the ith satellite

W1 L1 radian frequency $(2 \pi 1.57542 .109 \mathrm{rad} / \mathrm{s})$.

The SPS signal is BPSK (1) modulated on L5 and S bands. The navigation data at data rate of 50sps (1/2 rate FEC encoded) is modulo 2 added to PRN code chipped at 1.023Mcps identified for SPS service. The CDMA modulated code, modulates the $\mathrm{L} 5$ and $\mathrm{S}$ carriers at $1176.45 \mathrm{MHz}$ and $2492.028 \mathrm{MHz}$ respectively.

\section{C/A CODE GENERATION}

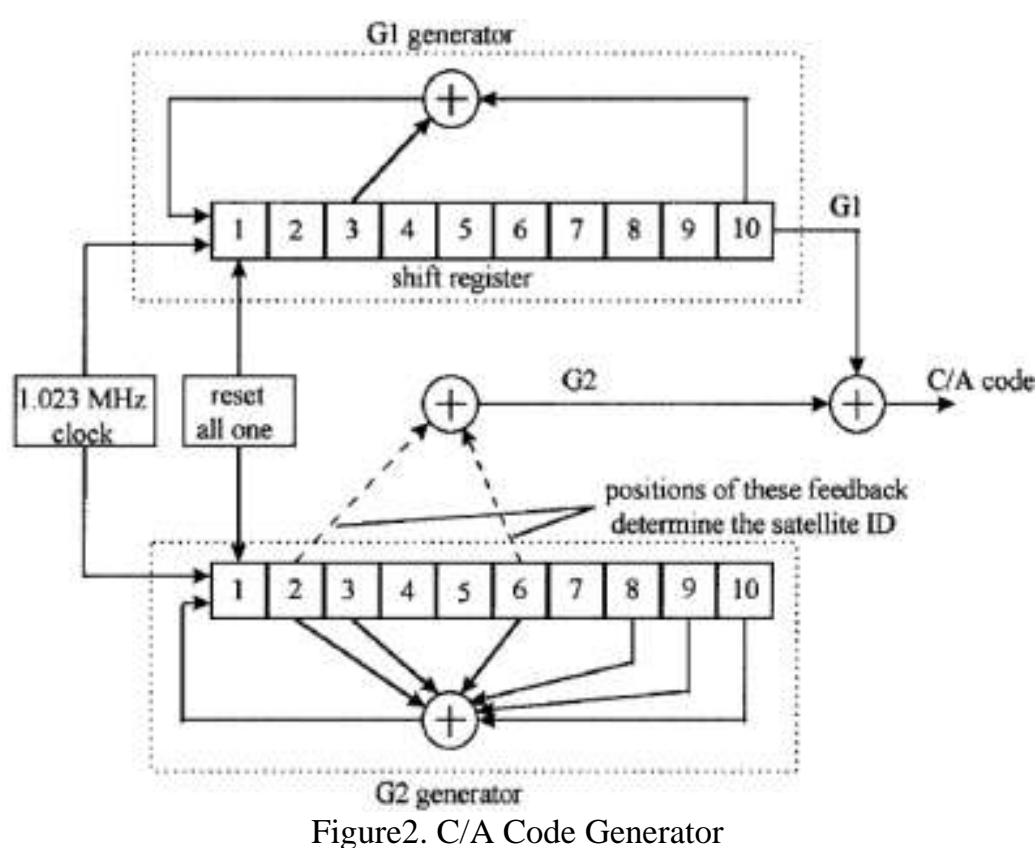

Each satellite uses a unique C/A code to implement the CDMA technique. The C/A codes belong to a family of Gold Pseudorandom Noise (PRN) codes. Gold codes are used because of their autocorrelation and cross-correlation properties. The architecture of a C/A code generator is shown in figure. Two-bit Linear Feedback Shift Registers (LFSR), G1 and G2, generate maximum length pseudo random codes with a length of $2^{\wedge} 10-1=1023$ bits. $\mathrm{N}$ is chosen to be 10 because PRN code has the data rate of 1023 chips per msec. Initially, both G1 and G2 are all set to ones, since the all-zero state is illegal. The G1and G2 LFSRs feedback taps are defined by the generator polynomials

$$
\begin{aligned}
& \mathrm{G} 1(\mathrm{X})=1+\mathrm{X} 3+\mathrm{X} 10 \\
& \mathrm{G} 2(\mathrm{X})=1+\mathrm{X} 2+\mathrm{X} 3+\mathrm{X} 6+\mathrm{X} 8+\mathrm{X} 9+\mathrm{X} 10
\end{aligned}
$$

The specific C/A code is generated by taking the exclusive-or output of the G1 LFSR and a delayed version of the output from the G2 LFSR. The delayed effect for the G2 LFSR output is obtained by the exclusive-or of two selected stages from the G2 LFSR. This will work since adding a phase shifted version of a PRN sequence to itself will shift the phase of the code, while not changing the code. 
The autocorrelation function of a C/A code is

$$
\operatorname{RCA}(\tau)=\int_{-\infty}^{\infty} C A i(t) C A i(t+\tau) d t
$$

where $\mathrm{CAi}$ is the $\mathrm{C} / \mathrm{A}$ code for the ith satellite and $\tau$ is the phase of the time shift. A normalized and simplified autocorrelation function of a typical C/A code is shown in figure 3.

The correlation peaks repeat every code period and the correlation interval is two chips. As mentioned this is a simplified model of the C/A code autocorrelation function since there are shifts which produce non-zero values between correlation peaks. The properties of the autocorrelation function is used to synchronize the receiver replicated code with the received code. It is important that the cross-correlation of any two C/A codes are minimal for any phase or Doppler shift over the entire code period. The ideal cross-correlation is defined by

$$
\operatorname{Rij}(\tau)=\int_{-\infty}^{\infty} \operatorname{CAi}(t) \operatorname{CAj}(t+\tau) d t=0
$$

where $\mathrm{CAi}$ is the $\mathrm{C} / \mathrm{A}$ code for the ith satellite and CAj C/A code for the $\mathrm{jth}$ satellite where $\mathrm{i \neq j}$. Due to the fact that multiple satellites signal is received with different delays and Doppler offsets the cross-correlation properties of C/A codes are not ideal, and may under certain conditions cause false acquisitions.

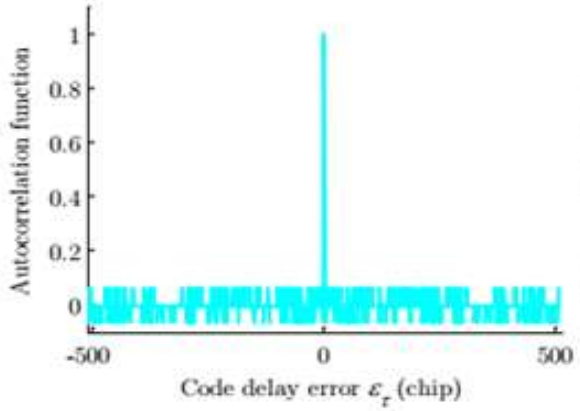

(a) Full function

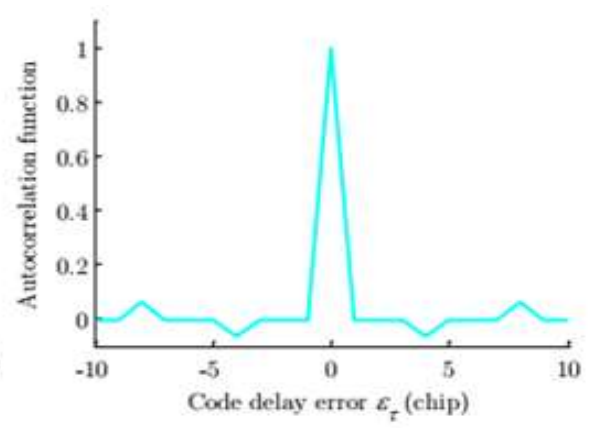

(b) Zoom around $\tau=0$

Figure 3. Normalized and simplified autocorrelation function of a typical C/A code

Above figure depicts the autocorrelation of the two signals which are shifted from 0 to 1023 bits. The output of the autocorrelation is maximum when shift in the second signal is minimum and it is at its peak when the shifted signal exactly matches the original signal.

\section{SIGNAL ACQUISITION}

Serial search is the simplest and most frequently used acquisition algorithm. A non-coherent correlator, shown in figure is used since the phase of the received signal is random. First the digital IF, $x$ (n), is multiplied by the replicated CA code, CA $[n+m]$. Here $n$ represents the nth sample and $m$ represents the number of samples the replicated CA code is phase shifted. Since we have a sampling rate of $5 \mathrm{MHz}$ the length, L, of one C/A code period is 5000 samples nominally.

After the code removal the in-phase (I) and quadrature (Q) components are generated. The I and Q components are accumulated for one or more code periods, N. The accumulated sum is squared. Next $\mathrm{K}$ correlations are accumulated to produce an averaged correlation point.

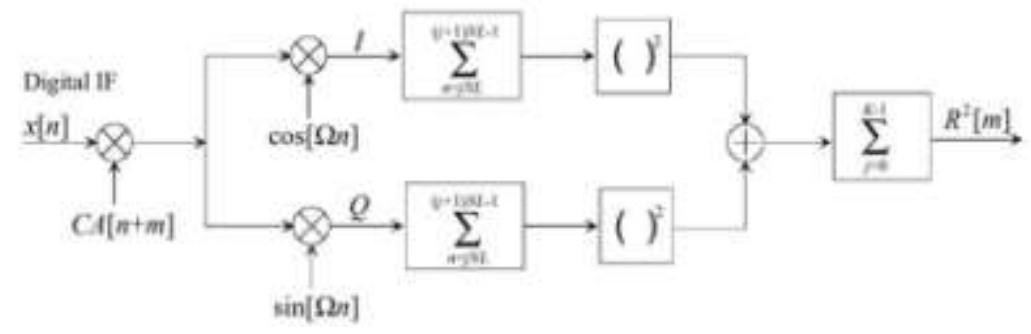

Figure 4. Non- coherent correlator in the domain 
If the correlation point is larger than a certain threshold it is assumed that the satellite is acquired. The correlation is approximately by the discrete sum:

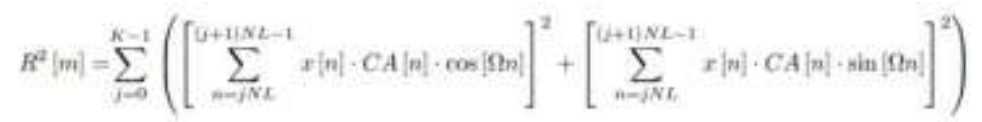

where $\Omega$ is radian frequency.

In MATLAB implementation, $\mathrm{N}=1$ and $\mathrm{K}=1$, since this provides the fastest acquisition. However choosing larger values for $\mathrm{N}$ and $\mathrm{K}$ will improve the acquisition of weak satellite signals and will also reduce the probability of false acquisition. One problem for choosing larger values of $\mathrm{N}$ is that it is possible that a data signal transition can occur during the correlation process. Choosing a larger $\mathrm{N}$ will however improve the signal to noise ratio since the accumulation is a low pass process. The data transitions are synchronized to the code periods and can occur at maximum every 20th code period. If $\mathrm{N}$ is even it is possible that a data bit transition will cause the dumped sum to be only noise. Choosing K>1 will not be sensitive to data bit transition, but he expected value of noise will not be zero since the noise is squared. This must be considered when the detection threshold is selected.

Usually when serial search is performed the code phase is incremented $1 / 2$ chip when one code has been tested. So there are 2 x $1023=2046$ code phases that must be tested. The MATLAB implementation however, shifts the code phase by one sample between each dump. One sample corresponds to approximately $1 / 5$ chip so here 5000 code phases are tested. 10000 samples of received data is correlated by sliding the replicated code over the 10000 samples, as illustrated in the figure.

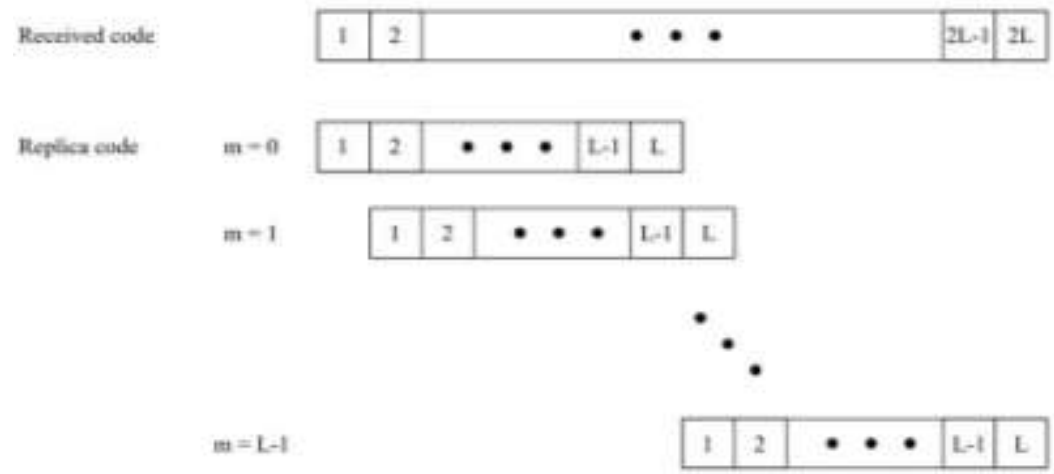

Figure 5. Serial Search Algorithm

Complicating things, the Doppler effects on the carrier must be taken into account. For a stationary receiver it is common to assume a $10 \mathrm{kHz}$ Doppler offset on the carrier. The acquisition process for each code period is stepped in the Doppler range in $500 \mathrm{~Hz}$ frequency increments. Choosing $500 \mathrm{~Hz}$ steps is a compromise in accuracy and speed. If the step was $1 \mathrm{kHz}$ there would be less frequency bins to test, but with the possibility that the residual Carrier of the I and Q components can cancel out the correlation, since $1 \mathrm{kHz}$ corresponds to the code period of $1 \mathrm{~ms}$.
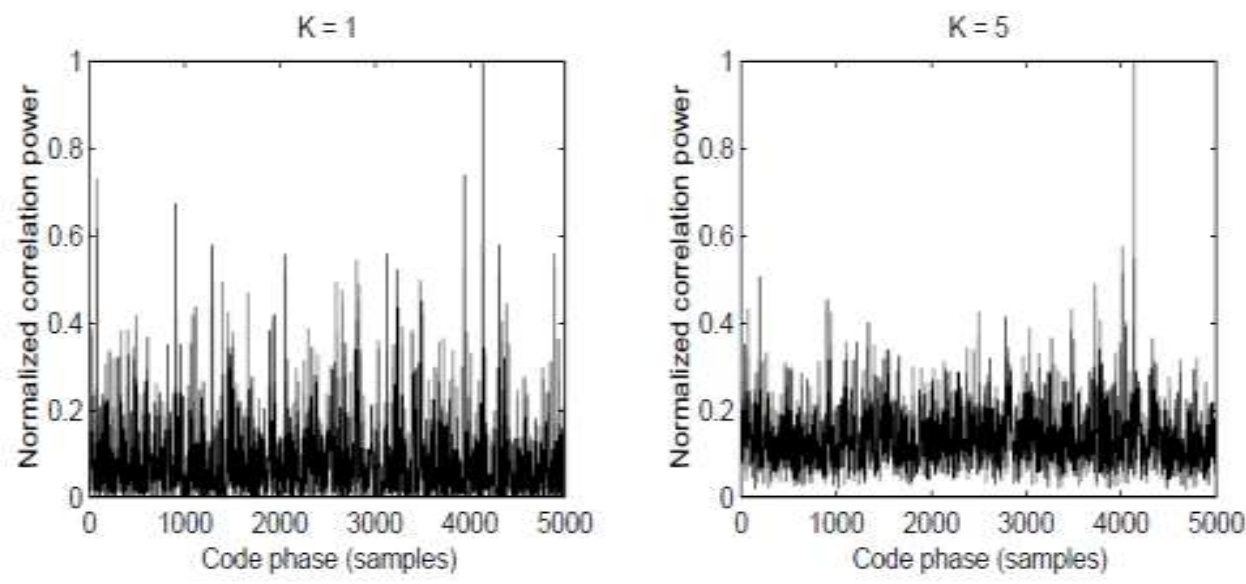
Vol. 8, Issue 4, April 2019
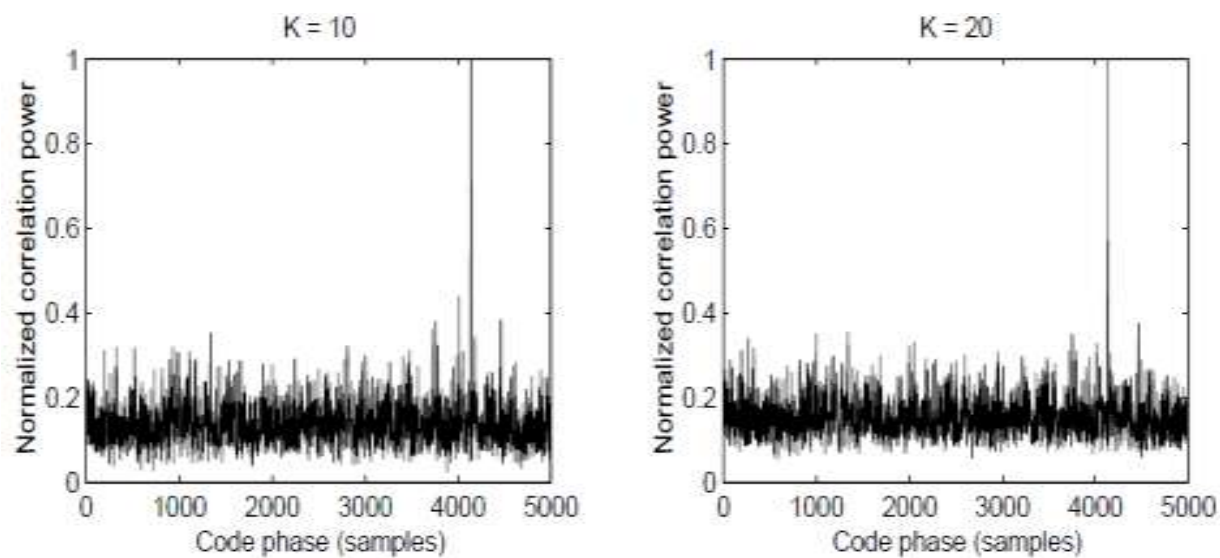

Figure 6. Acquisition of satellite at Intermediate frequency

\section{SIGNAL TRACKING}

Once satellite signals are detected in signal acquisition stage, continuous tracking of the satellite signal is equally significant for further decoding and extraction of navigation data and is referred to as signal tracking. The need for signal tracking arises from the fact the carrier signal experiences frequency deviation due to Doppler shift. Relative motion between satellite and receiver induces Doppler frequency offset in the carrier frequency and for stationary receiver it is assumed to be $\pm 10 \mathrm{KHz}$. Similarly C/A code phase samples also undergo shift.

Signal tracking is about detecting frequency and code shift of the detected satellite signal and synchronize with the replicated signals with the aim of retrieving navigation data. This is accomplished in two stages

1. Carrier tracking loop

2. Code tracking loop

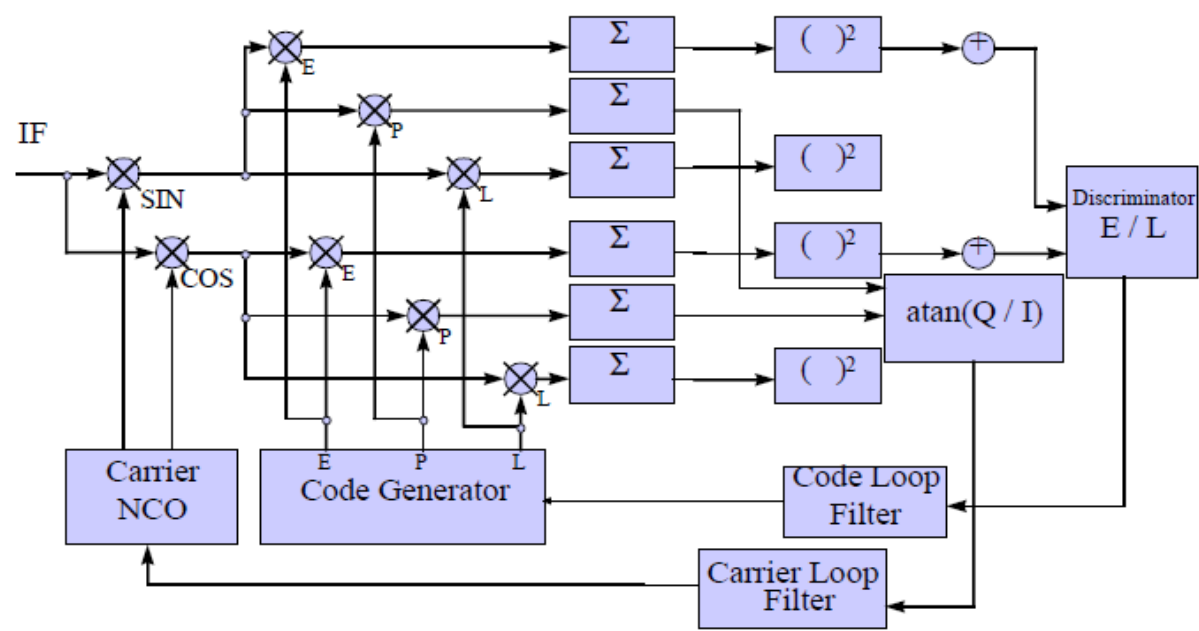

Figure 7. Block diagram for tracking

\section{Carrier tracking loop:}

Carrier tracking loop tracks the frequency and phase of the received signal by detecting the phase error between replicated signal and incoming signal and accordingly replicated signal produced by Numerically Controlled Oscillator (NCO) is adjusted to synchronize with incoming signal in both frequency and phase. For zero phase error navigation data is extracted.

\section{Code tracking loop:}

Post the carrier signal synchronization, received CA code samples is synchronized by aligning with replicated CA code samples by shifting right or left. To determine the direction of shift, the I and Q outputs are multiplied with prompt code (PRN code which is phase aligned), early code (prompt PRN code shifted by some samples to the right) and late code (prompt PRN code shifted by some samples to the left) resulting in corresponding to I and Q channel respectively. A code discriminator function is given by equation, generates error ( proportional to the code phase error between the 
replica and incoming signal. This error is filtered and applied to code generator and output frequency is increased or decreased and accordingly the prompt code is phase shifted to be phase aligned with received one.

A code discriminator is given by

$$
\varepsilon=\frac{\left(I_{E}^{2}+Q_{E}^{2}\right)-\left(I_{L}^{2}+Q_{L}^{2}\right)}{\left(I_{E}^{2}+Q_{E}^{2}\right)+\left(I_{L}^{2}+Q_{L}^{2}\right)}
$$

The above two loops function in sequence and satellite signal is tracked and navigation data is extracted.

MATLAB implementation of signal tracking :

$\mathrm{NCO}$ of the Carrier tracking loop and code generator of the code tracking loop is initialized to carrier frequency and code phase shift respectively, acquired from signal acquisition stage. The loop runs updating the NCO and code generator at every Integration time $(1 \mathrm{~ms})$ based on the discriminator output, remaining phase lock onto the signal, simultaneously retrieving the data.

\section{Performance analysis of carrier tracking loop:}

Performance of carrier tracking loop is measured in terms of ability of the loop to maintain phase lock onto the received signal and capability of tracking high signal dynamics. The carrier tracking loop tries to maintain phase lock of the signal as long as phase errors are within boundary. The dominant source of phase error in Costas PLL based receiver is thermal noise phase jitter and dynamic stress error. In order to maintain phase lock, the total phase error of the carrier tracking loop must not exceed one fourth the phase pull in range of arctangent discriminator of Costas loop.

\section{CONCLUSION}

The Indian Regional Navigation System is a complex system, which offers many different signal processing areas of research. In this paper we have limited the work to only a small subset of GPS which is the acquisition and tracking of GPS satellite signals using software. Generation of C/A code for different satellites is briefly discussed. Navigation data which contains the information of satellites position and time is then encoded on to the C/A code by modulo- 2 addition. A carrier wave with a frequency of $1575.42 \mathrm{MHz}$ is generated for transmission of signal from satellite to the user. Navigation data encoded in the C/A code is then modulated to the carrier using BPSK modulation technique. This modulated signal is transmitted from the satellite to the user. Thus the modulated signal from the satellite is received at the user end through suitable RF front end. The received signal is then performed with several search operations such as serial search and FFT search to find the Doppler shift in frequency. This Doppler shift in frequency of the transmitted signal is analyzed for the tracking of the user position.

\section{REFERENCES}

[1]. Indian Regional Navigation Satellite System, Signal In Space ICD for Standard Positioning Service (Version 1.0, ISRO-IRNSS-ICD-SPS 1.0), Indian Space Research Organization, June 2014, available at: http://irnss.isro.gov.in

[2]. E. D. Kaplan, C. J. Hegarty (Eds.), "Understanding GPS: Principles and Applications", Artech House, 2nd Edition, 2006

[3]. Borre, D. Akos , A Software-Defined GPS and GALILEO Receiver - A Single Frequency Approach, Birkhauser Boston, 2006.

[4]. Fredrik Johansson, Rahman Mollaei, Jonas Thor, and Jorgen Uusitalo, "GPS satellite signal acquisition and tracking" Division of Signal Processing, Lulea University of Technology, Sweden, August 21, 1998.

[5]. Rishija Misra and Shubham Palod "Code and Carrier Tracking Loops for GPS C/A Code", International Journal of Pure and Applied Sciences and Technology.

[6]. James Ba, Yen Tsui, Fundamentals of Global Positioning System Receivers: A Software Approach, John Wiley \& Sons, 2000.

[7]. Falin Wu, and Kefei Zhang," GPS signal Acquisition and tracking using software GPS receiver”, IEEE, 2005.

[8]. Manjula T R, G Raju, "Comphrensive study of Linear Kalman filter Based Tracking techniques under Ionosphere scintillations", proc. RAEREST 2016, March 2016.

[9]. Sophia Y. Zheng, "Signal acquisition and tracking for a software GPS receiver", Thesis for Master Degree, Department of Electrical engineering, Blacksburg, Virginia,2005.

[10]. S. Thombre, M. Z. H. Bhuiyan, S. Söderholm, M. Kirkko-Jaakkola, L. Ruotsalainen, H. Kuusniemi, "Investigating the Indian Regional Navigation Satellite System using a Software Multi- GNSS Receiver in Europe ", IETE, 2016. 